\title{
BP-PP-3-5
}

\section{Predictive value of metabolic activity detected by preoperative 18-fluorodeoxyglucose positron emission tomography/computed tomography in ampullary adenocarcinoma}

\author{
Youngmok PARK, Hyungil SEO*, Jaeri KIM, Myunghee YOON
}

Department of Surgery, Pusan National University Hospital, Busan, Korea

Introduction: In ampullary adenocarcinoma cases, the clinical effects of 18F-fluorodeoxyglucose (FDG) positron emission tomography/computed tomography (PET/CT) have not yet been well-studied, unlike other prognostic factors that have been reported till date. This study aimed to investigate the clinical impact of maximum standardized uptake value (SUVmax) in predicting the prognosis of ampullary adenocarcinoma.

Methods: Thirty-eight patients who underwent preoperative 18F-FDG PET/CT and curative-intent resection of ampullary adenocarcinoma at Pusan National University Hospital (Busan, Korea) between 2008 and 2017 were retrospectively analyzed in this study. We evaluated the clinicopathologic outcomes according to the SUVmax using univariate and multivariate Cox proportional hazard regression analyses and receiver operating characteristic analysis to arrive at a cutoff value.

Results: Lymph node metastasis was detected in nine patients, and 15 patients experienced a recurrence during the follow-up period. Among 38 patients, 33 showed an increased FDG uptake by the main tumor. SUVmax of 4.55 (sensitivity 61.5\% and specificity 60.0\%) was selected as a significant predictive factor for advanced lesions (larger size, $p=0.02$; advanced T stage, $p=0.02$; node metastasis, $p$ $=0.01$; and lymphovascular invasion, $p=0.04)$. Patients with SUVmax under 4.55 exhibited significantly longer overall survival than the rest $(<4.55$ vs. $\geq 4.55)$, and the 5 -year overall survival was $82.8 \%$ vs. $57.4 \%(p=0.05)$.

Conclusions: SUVmax of 4.55 on 18F-FDG-PET/CT could be a predictive factor for tumor biology and long-term survival in patients with ampullary adenocarcinoma. Nevertheless, considering the cost aspect and its limited prognostic effect, this study seems to require more patients. 